\title{
A Note on A-C Effects on MHD Dynamo in the Earth's Low-Latitude Magnetospheric Boundary Layer
}

\author{
Senkichi SHIBUYA \\ Faculty of Science, Yamagata University, Yamagata 990, Japan
}

(Received July 26, 1989; Revised September 28, 1990)

The interaction of the solar wind plasma flow with the earth's magnetopause boundary is far from a simple lamina flow and thus we can expect time variation of the flow velocity. When the turbulent plasma flow is able to cross the magnetic field due to the existence of local electric fields of the intense plasma waves, the fluctuating components of the solar wind plasma flow experiences a fluctuating electric field. Considering this, we have proposed an A-C dynamo model in the low-latitude magnetospheric boundary layer as well as estimated the effect of the induced alternating currents under a crossing type model to be described in the present paper.

The solar wind-magnetosphere dynamos have been proposed and discussed at different positions in the magnetosphere considering various models (COLEMAN, 1970; AKASOFU and KAN, 1973; AKASOFU, 1974, 1981; EASTMAN et al., 1976; ROSTOKER and Boström, 1976; Hones, 1979; PIDDINGTON, 1979; SonNERUP, 1980; Lundin, 1984).

At the magnetopause boundary, usually, the dynamo effects are considered under the frozen-in condition where the electric field $\boldsymbol{E}$ is given by $\boldsymbol{E}=-\boldsymbol{V} \times \boldsymbol{B}$, and both positive and negative particles drift perpendicular to $\boldsymbol{B}$ with the velocity $\boldsymbol{V}=\boldsymbol{E} \times \boldsymbol{B} / \boldsymbol{B}^{2}$ in the presence of the electric field $\boldsymbol{E}$ as considered by SONNERUP (1980) in the low-latitude plasma boundary layer (see Fig. 1). In the equatorial plane, the magnetopause is located at $y=0$.

The present model has therefore a unique point because it is assumed that the plasma makes drifts across the sheath magnetic field. The model is called "a crossing type dynamo" because the particle moves crossing the magnetic field; i.e. the particles experience $\boldsymbol{E}=\boldsymbol{V} \times \boldsymbol{B}$ field. In this model, however, we only considered the time varying component of $\boldsymbol{V}$, therefore, we are not concerning with the quasi-DC phenomena.

When irregularities in the solar wind plasma are sufficiently high, electric fields and plasmas of the magnetosheath can penetrate directly into the magnetosphere violating the frozen-in condition (e.g. LUNDIN and APARICIO, 1982; LUNDIN and DUBININ, 1985). In this turbulent conditions, the flow velocities of the penetrating hot plasma fluctuate in the boundary layer in both, space-wise and time-wise. Hence, we can expect an influence of an A.C. component in the turbulent magnetospheric boundary layer. We considered a simple magnetic-field crossing type model here, and calculated the influence of the time varying plasma flow whose effect can be described as an A.C. generator model. The considerations are made for the plasma flow in the low latitude boundary layer in the dawn and dusk flank sides.

As shown in Fig. 2, our magnetic field crossing type dynamo model is based on Sonnerup's model nevertheless, his model is related to the frozen-in type model (1980). 


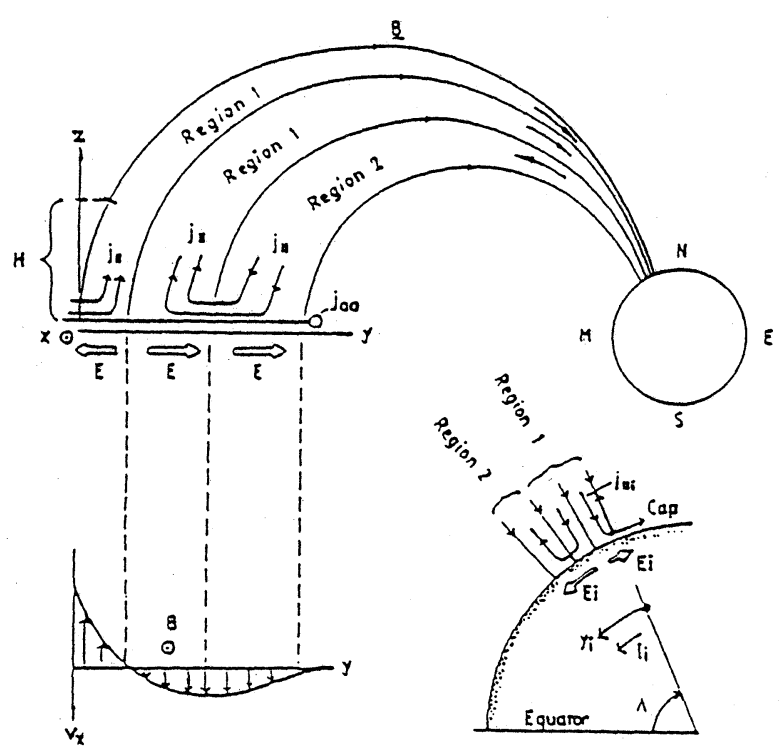

Fig. 1. View from the sun of the morningside boundary layer and its coupling to the ionosphere. Regions 1 and 2 correspond to the field-aligned current regions of Iijima and Potemra (after SonNERUP, 1980).

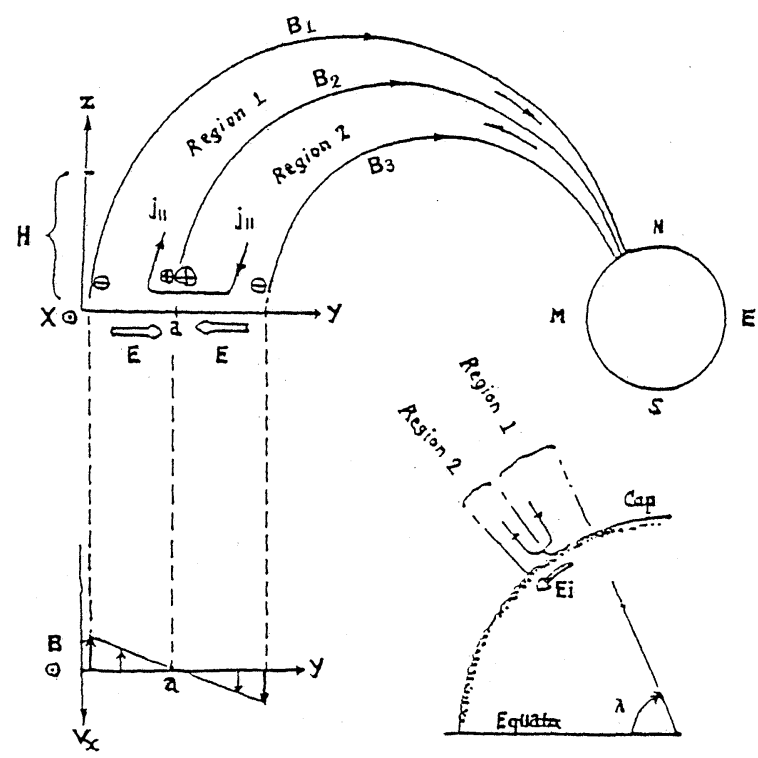

Fig. 2. A crossing type model for D-C and A-C generators in the morningside low-latitude boundary layer. 
The coordinates $(x, y, z)$ were chosen to coincide with those of the usual geocentric solar magnetospheric system. In our model, however, the electromotive forces are induced in the situation of the magnetic field crossing. The electric field vector showed different direction as compared to the field vector expected from the Sonnerup's frozen-in type model (cf. Fig. 1).

The flow direction of the solar wind plasma in the boundary layer (BL) is tailward $\left(\boldsymbol{V}_{x}<0\right)$; in the outside region, the returning sunward flow (RF) $\left(\boldsymbol{V}_{x}>0\right)$ exists adjacent to the boundary layer forming some sort of eddy convections. In the present model, the expression of the plasma flow distribution in the boundary layer is given by,

$$
\boldsymbol{V}_{x}=\left(\frac{y-a}{a}\right) \boldsymbol{V}_{0 x}
$$

where " $a$ " is the half width of the boundary layer (see Fig. 2).

If the magnetic field $\boldsymbol{B}$ in the magnetospheric boundary layer is uniform, the electromotive force $\boldsymbol{E}^{\mathrm{e}}$ is given by

$$
\boldsymbol{E}^{\mathrm{e}}=\boldsymbol{V}(y) \times \boldsymbol{B}
$$

where the bulk plasma velocity $\boldsymbol{V}(y)$ varies in proportion to $y$. Substituting, Eq. (1) into Eq. (2) we have

$$
\boldsymbol{E}_{y}^{\mathrm{e}}=-\left(\frac{y-a}{a}\right) V_{0 x} B_{z} \hat{y}
$$

where $\hat{y}$ is the unit vector along the $y$ axis (see Fig. 2). The directions of the electromotive force $\boldsymbol{E}^{\mathrm{e}}$ at the BL and RF in the dawn side are shown in Fig. 2.

Since the electromotive force $\boldsymbol{E}_{y}^{\mathrm{e}}$ is directed towards the point $y=a$, the ions are collected around the region $y=a$; and the electrons are concentrated at both ends $y=0$, and $2 a$. In the dusk side, the magnetic field vector $\boldsymbol{B}$ is directed in the same direction; the axis $y$ vector is defined towards the earth, to be in the opposite relation for $\boldsymbol{V} \times \boldsymbol{B}$. Hence negative charges accumulate at the center of the evening boundary layer (cf. Fig. 1 of SATO, 1982).

Even though the model setting is completely different, we can use the quantities following SONNERUP (1980); i.e. we take $V_{0 x}=200 \mathrm{~km} / \mathrm{sec}, B_{z}=30 \gamma=3 \times 10^{-8} \mathrm{wb} / \mathrm{m}^{2}$. Then from Eq. (3), it follows that

$$
\boldsymbol{E}_{y}^{\mathrm{e}}=-6\left(\frac{y-a}{a}\right) \hat{y} \quad \text { Volt } / \mathrm{km}
$$

The electrical potential reaches a maximum value at $y=a$ giving the result $V^{\mathrm{e}}=\int_{0}^{a} E_{y}^{\mathrm{e}} \mathrm{d} y=3 a$ Volt. The thickness " $a$ " of the BL-region is taken as $a=5000 \mathrm{~km}$ (e.g. EASTMAN and HONES, 1979). This thickness of the boundary regions can also be understood as a characteristic length of the region of the viscous interaction. For this thickness, we find the total voltage $V^{\mathrm{e}}=15 \mathrm{kV}$, which can be compared with a value anticipated by BOSTRÖM (1974). It appears that the positive ions concentrated to the vortex center 
$(y=a)$ by the electric field $\boldsymbol{E}_{y}^{\mathrm{e}}$ move down into the ionosphere along the magnetic field lines.

Since the effective specific conductivity $\sigma$ in the magnetosphere lies normally within a range in the order of $6 \times 10^{-8} \mathrm{mho} / \mathrm{m}$ (SONNERUP, 1980) for homogeneous and isotropic plasma, we can determine the electric current in the boundary layer as:

$$
\boldsymbol{j}_{0 y}=\sigma \boldsymbol{E}_{y}^{\mathrm{e}}=3.6 \times 10^{-10}\left(\frac{y-a}{a}\right) \quad \mathrm{A} / \mathrm{m}^{2} .
$$

It was found that this value is fairly comparable with the results so far obtained (cf. SHIBUYA, 1988) and that $\boldsymbol{j}_{0 y}$ flows toward the point $y=a$ on the equatorial plane.

WILLIAMS (1980) has indicated the presence of regular variations in the magnetopause orientation angle, which are consistent with boundary waves with amplitudes of a few hundred kilometers in the 150 - to 400 -s period range. Besides, it is well known by observations that the flow speed in the magnetospheric boundary layer is highly time variable (e.g. EASTMAN and HONES, 1979; SCKOPKE et al., 1981). Based on these evidences, we considered the quasi-uniform magnetic field $\boldsymbol{B}$ with the disturbed plasma flow velocity $\boldsymbol{V}(y, t)$ which is varying in time. We considered a case where the perturbation velocity $\boldsymbol{v}_{1}(y)$ varied sinusoidally in time flowing along the $X$ axis as a vector sum of steady speed $\boldsymbol{V}_{0}$ and perturbed component;

$$
\boldsymbol{V}_{x}=\boldsymbol{V}_{0 x}+v_{1} \cos (2 \pi f t) \text {. }
$$

Substituting this variable velocity into Eq. (2), we obtained the electromotive force

$$
E_{y}^{\mathrm{e}}=-V_{0 x} B_{z}-v_{1} B_{z} \cos (2 \pi f t) \text {. }
$$

If the frequency of the variable current is lower than the characteristic frequency $f_{0}$ $\left(=3.3 \times 10^{-3} 1 / \mathrm{sec}\right.$, cf. WILLIAMS, 1980) of the boundary layer oscillation, the electric current density $\boldsymbol{I}$ is determined directly by the e.m.f., $\boldsymbol{E}^{\mathrm{e}}$ with electrical conductivity $\sigma$, as

$$
\boldsymbol{I}=\sigma \boldsymbol{E}_{y}^{\mathrm{e}}=-\sigma V_{0 x} B_{z}-\sigma v_{1} B_{z} \cos (2 \pi f t)
$$

where the conductivity $\sigma$ includes the effective value that resulted by the wave particle interaction process at the boundary. In Fig. 3, the characteristic nature of the current is depicted with an arbitrary scale; we can see the DC component of the current overlapped with pulsating components.

Although there was no measurement of the perturbation speed separated from the steady speed in the boundary layer, the perturbation speed can be assumed to be much higher than one-tenth of the steady speed (e.g. LUNDIN and EVANS, 1985) and select normal conditions in the magnetospheric boundary layer, as $v_{1}=20 \mathrm{~km} / \mathrm{sec}, V_{0 x}=200$ $\mathrm{km} / \mathrm{sec}, B_{z}=30 \gamma, \sigma=6.8 \times 10^{-8} \mathrm{mho} / \mathrm{m}$ (cf. SONNERUP, 1980; SCKOPKE et al., 1981). Using these values, we can estimate the flowing current from Eq. (8) as

$$
I_{y}=(3.6+0.4 \cos 2 \pi f t) \times 10^{-10} \quad \mathrm{~A} / \mathrm{m}^{2} \text {. }
$$




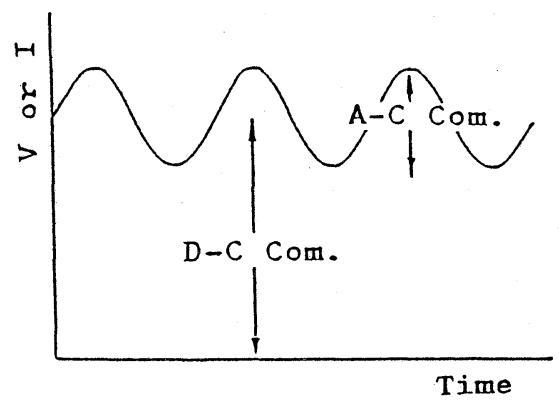

Fig. 3. Input and output are expressed in terms of superposition of D-C component and A-C component.

The "pulsating current" with density amplitude of $0.4 \times 10^{-10} \mathrm{~A} / \mathrm{m}^{2}$ is of interest in order to interpret the oscillating ULF geomagnetic pulsations in the connected ionosphere. The oscillating current can be observed as the oscillating magnetic field at the ionosphere level as well as on the ground. Considering the mechanism of this fluctuating component on the MHD dynamo we can understand the phenomena of the ULF pulsations as direct entry of hot solar wind plasmas into the polar ionosphere through the magnetic field lines.

\section{REFERENCES}

Akasofu, S.-I., The aurora and the magnetosphere: The Chapman memorial lecture, Planet. Space Sci., 22, 885-923, 1974.

AKasofu, S.-I., Magnetospheric substorms: A newly emerging model, Planet. Space Sci., 29, 1069-1078, 1981.

Akasofu, S.-I. and J. R. KAN, Some new thoughts on magnetospheric substorms, Radio Sci., 8, 1049-1057, 1973.

Boström, R., Ionosphere-magnetosphere coupling, in Magnetospheric Physics, edited by B. M. McCormac, pp. 45-59, D. Reidel, 1974.

Coleman, P. J., Jr., Tangential drag on the geomagnetic cavity, Cosmic Electrodyn., 1, 145-159, 1970.

Eastman, T. E. and E. W. Hones, Characteristics of the magnetospheric boundary layer and magnetopause layer as observed by Imp, J. Geophys. Res., 84, 2019-2028, 1979.

Eastman, T. E., E. W. Hones, Jr., S. J. BAme, and J. R. Asbridge, The magnetospheric boundary layer: Site of plasma, momentum and energy transfer from the magnetosheath into the magnetosphere, Geophys. Res. Lett., 3, 685-688, 1976.

HONEs, E. W., Jr., Solar wind-magnetosphere-ionosphere coupling, in Solar-Terrestrial Influences on Weather and Climate, edited by B. M. McCormac and T. A. Seliga, pp. 83-100, D. Reidel, Dordrecht, Holland, 1979.

Lundin, R., Solar wind energy transfer regions inside the dayside magnetosphere-2. Evidence for an MHD generator process, Planet. Space Sci., 32, 757-770, 1984.

Lundin, R. and B. APARICIO, Observation of penetrated solar wind plasma elements in the plasma mantle, Planet. Space Sci., 30, 81-91, 1982.

Lundin, R. and D. S. Evans, Boundary layer plasmas as a source for high-latitude, early afternoon, auroral arcs, Planet. Space Sci., 32, 1389-1406, 1985.

Lundin, R. and E. M. Dubinin, Solar wind energy transfer regions inside the dayside magnetopause: Accelerated heavy ions as tracers for MHD-processes in the dayside boundary layer, Planet. Space Sci., 33, 891-907, 1985.

Piddington, J. H., The closed model of the earth's magnetosphere, J. Geophys. Res., 84, 93-100, 1979.

Rostoker, G. and R. Boström, A mechanism for driving the gross birkeland current configuration in the auroral oval, J. Geophys. Res., 81, 235-244, 1976. 
Sato, T., Auroral physics, in Magnetospheric Plasma Physics, edited by A. Nishida, Chap. 4, D. Reidel, Boston, 1982.

Sckopke, N., G. Paschmann, G. Haerendel, B. U. Ö. Sonnerup, S. J. Bame, T. G. Forbes, E. W. Hones, Jr., and C. T. RusSEll, Structure of the low-latitude boundary layer, J. Geophys. Res., 86, 2099-2110, 1981.

SHibuYA, S., An estimate of the polarization current induced by the interaction of the solar wind plasma with the magnetotail boundary, J. Geomag. Geoelectr., 40, 357-365, 1988.

Sonnerup, B. U. Ö., Theory of the low-latitude boundary layer, J. Geophys. Res., 85, 2017-2026, 1980.

Williams, D. J., Magnetopause characteristics at 0840-1040 hours local time, J. Geophys. Res., 85, 3387-3395, 1980 . 